\title{
UNA VISIÓN ALTERNA DE LA FIRMA DESDE LA ORGANIZACIÓN, EL APRENDIZAJE Y LOS PARADIGMAS TECNOLÓGICOS
}

Juan Camilo Galvis-Ciro

Universidad Pontifica Bolivariana Colombia

María Alejandra Echavarría-Arcila Universidad Pontificia Bolivariana Colombia 
Panorama Económico, Vol. 26 - No. 4 (Octubre - Diciembre de 2018), pp. 481-496

Juan Camilo Galvis-Ciro

María Alejandra Echavarría-Arcila

\title{
Una visión alterna de la firma desde la organización, el aprendizaje y los paradigmas tecnológicos
}

\section{Resumen}

Este documento presenta una reflexión acerca de los conceptos de empresa y tecnología. Para ello, se discuten otras visiones de la empresa que difieren con la visión neoclásica, las cuales consideran el esquema organizativo, los paradigmas tecnológicos y el aprendizaje organizacional. Es evidente que los conceptos neoclásicos de empresa y de tecnología son limitados y no permiten comprender la dinámica de las firmas en las economías de mercado. Cómo resultado se propone una concepción alternativa de la tecnología y de la empresa, así como una nueva esquematización de la firma.

Palabras clave: Empresa; Tecnología; Esquema Organizativo; Paradigma Tecnológico; Aprendizaje Organizacional.

Clasificación JEL: D21, D23, D24.

\section{Another vision about firms from the organization, learning and technological paradigms.}

\begin{abstract}
This document presents a reflection on the concepts of business and technology. For this, other visions of the company that differ with the neoclassical vision are discussed, which consider the organizational scheme, technological paradigms and organizational learning. It is evident that the neoclassical concepts of business and technology are limited and do not allow understanding the dynamics of firms in market economies. As a result, an alternative conception of technology and business is proposed, as well as a new outlining of the firm.

Keywords: Technology; Organizational Scheme; Technological Paradigm; Organizational Learning. JEL Classification: D21, D23, D24.
\end{abstract}

\section{Une vision alternative de l'entreprise à partir des paradigmes organisationnels, d'apprentissage et technologiques}

\section{Résumé}

Ce document présente une réflexion sur les concepts de commerce et de technologie. Pour cela, d'autres visions de l'entreprise qui diffèrent de la vision néoclassique sont abordées, qui prennent en compte le schéma d'organisation, les paradigmes technologiques et l'apprentissage organisationnel. Il est évident que les concepts néoclassiques de commerce et de technologie sont limités et ne permettent pas de comprendre la dynamique des entreprises dans les économies de marché. En conséquence, une autre conception de la technologie et des affaires est proposée, ainsi qu'une nouvelle présentation de la société.

Mots-clés: La Technologie; Schéma D’organisation; Paradigme Technologique; Lapprentissage Organisationnel.

Nomenclature JEL: D21, D23, D24. 


\title{
Una visión alterna de la firma desde la organización, el aprendizaje y los paradigmas tecnológicos
}

Recepción de artículo: 21/05/2018

Concepto de evaluación: 30/06/2018

Aceptación de artículo: 05/08/2018

\author{
Juan Camilo Galvis-Ciro*
Universidad Pontifica Bolivariana \\ Juan Camilo Galvis-Ciro*
Universidad Pontifica Bolivariana \\ Colombia \\ María Alejandra Echavarría-Arcila \\ Universidad Pontificia Bolivariana \\ Colombia
}

\section{INTRODUCCIÓN}

La conceptualización de la firma y de la tecnología ha sido abordada desde diferentes perspectivas, con un alcance particular en cada caso. En la teoría económica, el modelo neoclásico propone la visión tradicional a este respecto, al definir la empresa como una 'caja' transformadora de insumos y la tecnología como una información pública sobre el proceso de producción (Arrow y Debreu, 1954). Sin embargo, esta concepción resulta limitada para dar cuenta de fenómenos relevantes en las economías de mercado, tales como el aprendizaje, el cambio técnico y la innovación.

Debidoa estas restricciones, otras teorías se han ocupado de explicar la firma y su tecnología desde diversos puntos de vista. Entre ellas, resultan relevantes las teorías relacionadas con el esquema organizativo (Penrose, 2009), los paradigmas tecnológicos (Dosi, 1988a,b) y el aprendizaje organizacional (Malerba, 1992). No obstante, estas aproximaciones han sido desarrolladas de manera aislada, lo cual conduce, una vez más, a limitar su capacidad explicativa frente a la complejidad de los fenómenos económicos.

En vista de lo anterior, el presente documento propone una visión alterna de la firma y de la tecnología, con el fin de superar las limitaciones de la teoría neoclásica y de conciliar la aparente disparidad y dispersión de otros conceptos relevantes. De esta manera, se pretende contribuir con una nueva aproximación conceptual más amplia e integral, que conjugue las distintas perspectivas ya mencionadas y permita una visión más robusta.

\footnotetext{
* Autor para correspondencia 
Este documento está organizado como sigue. En la segunda sección es presentada la teoría neoclásica de la empresa y sus limitaciones. A continuación, en la tercera sección, es discutida la importancia del esquema organizativo para la empresa. En la cuarta sección, es analizado el concepto de paradigma tecnológico y sus implicaciones. Luego, en la quinta sección son presentados los aprendizajes que toman lugar en la empresa y es propuesto un esquema que pretende solucionar las limitaciones de la teoría neoclásica y busca conjugar las perspectivas estudiadas. Por último, son presentadas las conclusiones.

\section{LA TECNOLOGÍA Y LA FIRMA EN LA ECONOMÍA NEOCLÁSICA}

La teoría neoclásica en economía se refiere al modelo de equilibrio general competitivo desarrollado por Kenneth Arrow y Gerard Debreu (1954), quienes proponen una visión particular para analizar la conducta de la empresa y los consumidores en las economías de mercado.

La firma o la empresa para la teoría neoclásica es una abstracción tanto de las formas legales de organización, como sobre el sector económico al que pertenece (Monsalve,1999). En particular, la tecnología es el conocimiento que tienen las empresas para combinar insumos. Este conocimiento se concibe como una información codificada, clara y explícita que llega a la empresa de forma exógena. Es decir, la tecnología tiene la característica de ser una información común, la cual está disponible en una reserva o stock de conocimiento público sin costos de adquisición y, por ende, no puede ser apropiada o protegida por una firma en particular (Dosi, 1982; Freeman, 1994).

La teoría neoclásica es formulada en un ambiente competitivo, en el cual no existe poder de mercado. Por tanto, todas las empresas tienen la misma información para llevar a cabo la producción, es decir, sobre cómo transformar los insumos en productos. Además de la mencionada simetría, existen otros dos supuestos. En primer lugar, se considera que la información es completa, ya que se conocen los precios de todos los bienes y las consecuencias de todas las acciones. En segundo lugar, se asume que la información es perfecta, ya que todas las empresas conocen la información al mismo tiempo. En suma, la información es considerada como simétrica, completa y perfecta (Stiglitz, 2000).

Esta teoría postula también que el conjunto de posibilidades tecnológicas (el llamado conjunto de producción) tiene unas características particulares, tales como: posibilidad de inacción, combinación de tecnologías, aditividad e irreversibilidad (Varian, 1992). Bajo estos supuestos, el concepto neoclásico de tecnología adopta la forma de una función de producción, que expresa de forma precisa y exacta la relación entre varias combinaciones técnicamente factibles de insumos $\left(\mathrm{x}_{1}, \mathrm{x}_{2}, \ldots, \mathrm{x}_{\mathrm{n}}\right) \mathrm{y}$ productos $(\mathrm{y})$, en virtud del conocimiento técnico existente sobre este tipo de relaciones, esto es: la tecnología queda resumida en $\mathrm{y}=\mathrm{f}\left(\mathrm{x}_{1}, \mathrm{x}_{2}, \ldots \mathrm{x}_{\mathrm{n}}\right)$. Esta noción de la firma como una caja que produce transformaciones es presentada en la figura 1.

Cuando la tecnología adopta la forma de la función de producción, la economía neoclásica delimita de forma más precisa el concepto de tecnología junto con la empresa y, en consecuencia, la teoría pierde alcance. En particular, una vez que la producción se lleva 


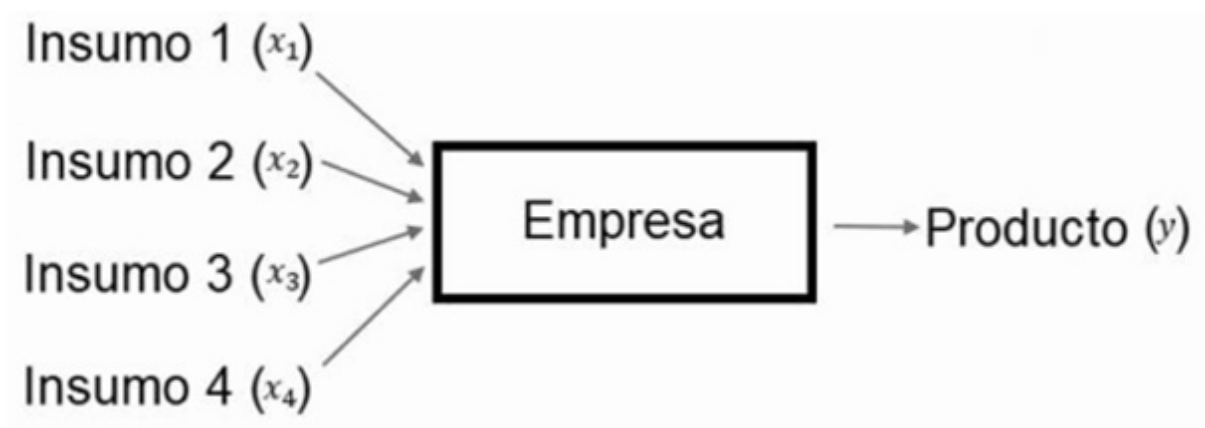

Figura 1. La empresa neoclásica. Elaboración propia.

a cabo de forma mecánica a través de una receta expresada en la función de producción, la empresa neoclásica queda reducida a una 'caja negra', en la cual entran insumos que luego salen transformados en productos dado que no se dice o se sabe algo acerca de la organización o los procesos que toman lugar allí (Dosi, 1982).

La teoría neoclásica asume que los precios de los bienes están dados y que las empresas no tienen la capacidad de fijar los precios. Es decir, a unos precios exógenos y conocidos la empresa sólo debe encontrar las combinaciones de insumos que le maximicen el beneficio. Dicho en otros términos, el modelo asume una conducta paramétrica y pasiva en las empresas ya que estas toman una información conocida y buscan, de forma mecánica, demandar insumos y producir de forma óptima.

Varios de los supuestos hechos sobre la firma y la tecnología no son adecuados para el análisis de las firmas en economías de mercado. A lo sumo, la visión neoclásica de la tecnología sólo se corresponde con las actividades de producción más básicas o rudimentarias, en las cuales la producción no requiere conocimientos especializados difíciles de expresar y codificar o donde no es necesario un esquema organizativo.

Conforme puede observarse, la tecnología puede verse como una receta de cocina. Al respecto, Sala-i-Martin (2000) hace una ilustración bastante diciente sobre el concepto neoclásico de tecnología. Dicho autor comenta lo siguiente:

\begin{abstract}
"Para producir galletas es necesario tener capital (hornos, harina, huevos y otros materiales producidos previamente) y trabajo (cocineros). También es necesario saber cómo mezclar los ingredientes para llegar al producto final. Sin el conocimiento de la fórmula, es imposible producir galletas por más hornos, harina y cocineros que tengamos. Esa fórmula es lo que llamamos tecnología." (Sala-i-Martin 2000, p. 168)
\end{abstract}

La tecnología como una receta o una información públicamente conocida posee las características que tiene el conocimiento científico más elemental. Es decir, la tecnología es un bien no rival y no excluyente. La característica de ser no rival implica que el conocimiento necesario para llevar a cabo la transformación de insumos en productos por parte de una firma no se acaba o no se vuelve indisponible cuando alguna firma lo 
utiliza. Es decir, todas las firmas pueden utilizar al mismo tiempo el conocimiento para combinar insumos, ya que todas lo conocen y está disponible sin costo alguno (Sala-iMartin, 2000).

Con relación a la tecnología como un conocimiento no excluyente, en el modelo de competencia perfecta de Arrow y Debreu el supuesto es necesario para poder evitar que alguna empresa tenga poder de mercado mediante la exclusión en el uso de algún conocimiento con fines productivos. Es importante aclarar que la exclusión se puede dar de varias formas. Una de ellas sería que alguna empresa usará alguna patente para proteger el conocimiento que tiene para producir. No obstante, como el conocimiento se asume que es conocido por todas las empresas, disponible en un stock público, esta opción no tendría viabilidad. Otra opción sería que el conocimiento para producir fuera tan especializado que sólo algunas empresas pudieran procesarlo o supieran cómo utilizarlo, lo cual haría que ciertas empresas fueran más capacitadas que otras, algo que invalida el supuesto de un ambiente competitivo donde todas las firmas son iguales.

Es importante anotar que, desde los inicios del capitalismo, la tecnología ha sido un cuerpo de conocimientos y experiencias producido al interior de las empresas, con una dinámica difícil de esquematizar en una relación simple de insumos-productos (Nelson, 1988). Por lo tanto, la teoría neoclásica deja por fuera actividades de producción industriales, en las que la tecnología es un fenómeno complejo y difícil de reducir a una función de producción o expresar en una receta.

Esta teoría, al hacer abstracción de las formas de organización de las empresas, no comprende tampoco el papel que juegan otros elementos que también influyen en la producción, tales como: la organización, la cultura de trabajo y el ambiente laboral. Es decir, deja por fuera el conocimiento implícito de las empresas, para el cual no existen mercados, ya que esta información está protegida mediante varios mecanismos.

Debe agregarse además que la producción toma tiempo y aprendizajes. En particular, la producción de cualquier bien o servicio requiere habilidades especiales, entrenamientos avanzados en ciencia o ingeniería, trabajo colaborativo y uso de instrumentos o equipos costosos que requieren aprehensión en sus diversos componentes. Estos recursos no están disponibles de forma libre en el mercado y suelen encontrarse dentro de las empresas, dado que estas han encontrado un factor competitivo en las rutinas de producción (Nelson, 1988).

En suma, tanto la organización como los aprendizajes son recursos de las empresas que pueden no tener la característica de información libre, simétrica y perfecta. Es decir, el conocimiento tecnológico, sus fortalezas y debilidades, así como las necesidades del usuario, tienden a ser privados o apropiados y no disponibles para quienes no tienen una relación directa con la empresa (Nelson, 1988; Dosi, 1988b). En definitiva, el concepto neoclásico de tecnología y, por tanto, de empresa, son limitados, por lo que es necesario analizar desde otro punto de vista todo lo relacionado con la firma y la producción. 


\section{LA EMPRESA Y EL ESQUEMA ORGANIZATIVO}

Para colocar en contexto el planteamiento neoclásico que afirma que la tecnología es sólo información sobre cómo combinar los insumos, es relevante plantear la siguiente pregunta. Suponga que se tienen los insumos necesarios para producir un edificio: el acero, los ladrillos, el cemento, la mano de obra y demás elementos constructivos. Asuma que existe información codificada sobre cómo se combinan aquellos elementos para producir el edificio. ¿Por qué no cualquier grupo de personas podría ser capaz de producirlo?

Es posible enfocar la respuesta desde la importancia de la organización. Para cualquier actividad de producción es fundamental la organización, las experiencias y aprendizaje. Así exista la información sobre cómo combinar los insumos, esta no sirve de nada si no hay experiencias, organización y coordinación sobre el uso de los recursos. Así, en primera instancia, la firma puede y debe ser entendida como una organización administrativa de recursos productivos (Penrose, 2009).

La organización es necesaria para ejecutar cualquier plan de producción. Por lo general, las firmas se organizan por unidades y cada una administra diferentes recursos, las cuales a su vez son coordinadas desde una dirección centralizada. Por ejemplo, la unidad de recursos humanos se encarga de organizar la mano de obra y sus labores. La unidad de recursos físicos administra la maquinaria y el stock de insumos. La unidad de recursos financieros gerencia los pagos y compras de los insumos y la unidad de ventas gestiona los mercados del producto de la empresa. Todas estas unidades son coordinadas por un gerente general, el cual tiene como función hacer que todas las unidades lleven a cabo la producción de manera lucrativa para los dueños de la firma. Este esquema organizativo se representa en la figura 2.

Estructura organizativa

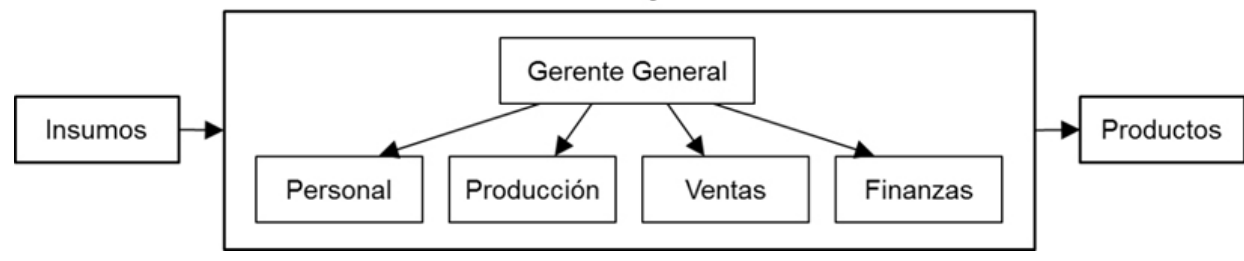

Figura 2. El esquema organizativo de la empresa. Elaboración propia.

La firma no es sólo una caja negra que combina insumos y que toma de decisiones relacionadas con la cantidad de producción dados los precios, ya que existen otros atributos que trascienden a la teoría neoclásica de la empresa, entre estos: el crecimiento y el aprendizaje (Penrose, 2009). En las economías de mercado, la empresa debe definirse como una organización cuya actividad económica se desarrolla dentro de un esquema organizativo que tiene ciertos desdoblamientos para articular cada vez mayores y variados recursos. En conjunto con esto, la empresa tiene una planificación consciente que direcciona el crecimiento, ya que toda actividad de producción requiere gestión. Es decir, una vez la firma es vista como una unidad administrativa, puede afirmarse que 
las empresas sí crecen y este fenómeno puede ser medido por medio de la cantidad de recursos productivos que configura y coloca en movimiento de una forma particular.

Por lo tanto, la empresa se puede considerar como una unidad de planificación administrativa autónoma y su principal función económica es tomar decisiones administrativas para disponer y hacer uso de los recursos productivos. El fin de la organización es suministrar bienes y servicios de acuerdo con los planes desarrollados y puestos en práctica dentro de la propia empresa (Penrose, 2009).

En la medida que se desarrolla la producción, cada unidad (de recursos humanos, de ventas, de producción, etc.) toma rasgos característicos y se moldea conforme a las experiencias. Es decir, cada unidad aprende en medio de la actividad misma de producción y adquiere experticias, por ejemplo, sobre la mejor forma de combinar insumos, los modos de incentivar los trabajadores y el mejor medio de llevar el producto al mercado. Este aprendizaje es único y propio y hace parte propia de la tecnología de la empresa.

En suma, de nada vale tener los insumos y la información sobre cómo estos se combinan si no existe un esquema organizativo. Por tanto, la tecnología de la empresa es dinámica e involucra organización, aprendizajes y rutinas, que en la mayor parte de los casos no se puede expresar en información pública, ya que sólo se adquieren de manera privada, una vez se inicia la producción.

\section{EL PARADIGMA TECNOLÓGICO Y EL SECTOR DE PRODUCCIÓN}

Además del esquema organizativo, cada tecnología tiene características propias que hacen imposible la generalización que propone la visión neoclásica de la firma. Para entender la importancia del sector de producción al momento de analizar la tecnología de la empresa, es importante analizar la teoría de los paradigmas tecnológicos. Este concepto fue construido a partir de un paralelo con los llamados paradigmas científicos, por lo que vale la pena recordar su significado.

Por paradigmas científicos se entiende el conjunto de prácticas y saberes que definen una disciplina científica. Estos saberes direccionan el estado de arte sobre algún fenómeno y, en particular, estructuran las diferentes ciencias (economía, física, matemáticas, derecho, etc.). Así, cada paradigma científico define los problemas, los métodos, los campos de investigación y las fronteras del conocimiento existente sobre algún fenómeno (Kuhn, 1970).

A partir de esto, el concepto de paradigma tecnológico puede ser definido como las técnicas y conocimientos existentes para llevar a cabo una actividad de producción. El paradigma tecnológico es un cuerpo específico de conocimientos que enfrenta cada empresa y que guía las actividades de búsqueda, desarrollo y mejoramiento de los procesos de producción (Dosi, 1988a,b).

Es posible establecer que la tecnología de una firma depende del paradigma tecnológico en el que se encuentra. Como este paradigma es resultado de las experiencias pasadas, los logros y el estado del arte actual para producir, se puede afirmar que la tecnología de cada 
firma tiene componentes históricos, locales e institucionales, sobre los cuales no se puede hacer abstracción, tal y como pretende la teoría neoclásica (Dosi, 1982).

Cada empresa enfrenta un paradigma tecnológico. Por ejemplo, las empresas del sector automovilístico enfrentan el paradigma de la máquina de combustión interna y la producción en cadenas de montaje. Ambos paradigmas definen y caracterizar la forma de llevar a cabo la producción de automóviles y direccionan las posibles mejoras que se puedan hacer. En este caso, las empresas productoras de automóviles se enfrentan a la necesidad de tener conocimientos en física, producción de energía, ensamblaje, termodinámica y otro conjunto de conocimientos particulares que caracterizan la producción de un automóvil. Este paradigma es diferente, por ejemplo, al paradigma que enfrentan las empresas que producen alimentos o vestidos.

El paradigma tecnológico puede entenderse entonces como un patrón o un programa de investigación para cada tecnología. Esto es, como un marco sobre las necesidades contextuales en un campo tecnológico (como un sector industrial, por ejemplo), al igual que como los modelos para resolver problemas dentro de este. En este sentido, el paradigma tecnológico es alterado a partir de las pruebas y errores tanto de individuos como de empresas, y a menudo es compartido por estos como la base para mejorar la eficiencia de los procesos y el rendimiento de los productos (Dosi, 1988b).

Es posible afirmar entonces que un paradigma tecnológico es un modelo y un patrón de solución a problemas de producción seleccionados, con base en principios científicos y en materiales específicos (Dosi, 1982). Por lo tanto, un régimen tecnológico define de manera contextual las necesidades que se pretenden satisfacer y las oportunidades que existen para modificar la tecnología, esto es, para innovar. Así mismo, establece los procedimientos, los principios y los conocimientos que se utilizan para hacerlo (Dosi, 1988b).

La creación de nuevos paradigmas tecnológicos es contextual al surgimiento de necesidades económicas (Dosi, 1982). No obstante, la forma en que se hace frente a las demandas del mercado depende de modos de búsqueda específicos, de ciertas bases de conocimiento y de ciertas combinaciones entre las formas de conocimiento tecnológico particular, privado y público, que cada paradigma tecnológico posee (Dosi, 1988a). Es decir, las firmas no responden a las necesidades del mercado cambiando la combinación de insumos de forma pasiva, tal y como asume la teoría neoclásica del cambio técnico (Sahal, 1981). Ante el cambio en las necesidades del mercado vía cambios en los precios, la respuesta de las firmas depende de forma crítica de las características del paradigma tecnológico en el que se encuentran. Por tanto, es importante definir los componentes de cada régimen tecnológico si se desean conocer cuáles pueden ser las direcciones del cambio técnico.

Varios elementos definen y modifican un paradigma tecnológico, los cuales son: la oportunidad tecnológica, la apropiabilidad y las condiciones de la demanda (Dosi, 1988b). El primer elemento define el conjunto de opciones para el avance técnico y las oportunidades de innovación que cada paradigma implica. Es decir, la oportunidad tecnológica hace referencia a la fertilidad económica y técnica de los esfuerzos que son 
necesarios para introducir una mejora en la tecnología. Por tanto, ella está relacionada con las posibles modificaciones en el proceso de producción o en los atributos del producto que se pueden lograr mediante inversiones en investigación y desarrollo (I+D). Esta fertilidad va a depender de forma significativa del sector al que pertenece cada firma, ya que el grado de dificultad para llevar a cabo la producción depende del tipo de bien y de la incorporación de conocimiento que este posea.

La oportunidad tecnológica es alta o baja dependiendo de la concentración geográfica de las empresas, de sus conocimientos específicos, de sus fronteras de conocimiento y de sus procesos de aprendizaje. Así, estas oportunidades son locales y específicas para cada empresa y se definen tanto por la facilidad para lograr avances tecnológicos, como por los diferentes grados de acumulación de tales avances a nivel de industria o de empresas individuales (Dosi, 1988a,b). La oportunidad tecnológica es entonces diferente en empresas que producen bienes como alimentos (que incorporan conocimientos bastante genéricos en agricultura, composición física, ingredientes químicos y energía), con relación, por ejemplo, a empresas que producen computadoras (que incorporan conocimientos avanzados en microprocesadores, óptica y electrónica). Ambas empresas, además, requieren esquemas de organización diferentes para llevar a cabo la producción.

Ciertos factores contribuyen o afectan la oportunidad tecnológica que enfrenta cada paradigma y, en consecuencia, cada empresa. Un primer factor son los avances científicos, los cuales abren nuevos entendimientos y traen solución a viejos problemas. Estos avances expanden el stock de datos, técnicas y capacidades para solucionar los problemas. En consecuencia, las empresas pueden diferir en su oportunidad tecnológica en función de qué tan lejos o no estén de las fronteras que abren los nuevos conocimientos. Un segundo factor son los gastos en I+D a nivel de empresa y de país, los cuales son necesarios para explotar las oportunidades tecnológicas, ya que estas requieren tiempos y recursos para entender las posibles rutas de avance. El último lugar están los feedbacks de experiencias pasadas y los avances de otras industrias, los cuales son importantes para direccionar los esfuerzos en ciertas trayectorias (Dosi, 1988b; Heijs y Buesa, 2016).

El segundo elemento de un paradigma tecnológico son las condiciones de apropiabilidad. Estas aluden a la posibilidad de obtener un derecho de propiedad sobre las posibles mejoras en una tecnología, lo cual determina los retornos de la inversión en I+D. Es decir, la oportunidad tecnológica va a ser explotada en la medida que se puedan establecer derechos de propiedad sobre las mejoras.

La apropiabilidad refleja las posibilidades que tiene la firma de proteger las mejoras en la tecnología de la imitación y da cuenta de los diferentes grados en que las empresas pueden obtener rendimientos económicos de varios tipos de mejoras. Por ende, la apropiación depende del alcance del sistema de propiedad intelectual; de los costos y el tiempo necesarios para la duplicación; de los efectos de la curva de aprendizaje, y de los esfuerzos en ventas y servicio (Dosi, 1988a). Por lo general, cada tecnología involucra diferentes condiciones de apropiabilidad y aquellas que requieren conocimientos más especializados, disfrutan de una ventaja en la protección en su tecnología, ya que no cualquier empresa o individuo puede conocer la forma de producción. 
Cuando la apropiabilidad es baja, es más fácil duplicar las modificaciones en la tecnología que hacen ciertas firmas, lo cual disminuye los incentivos para realizar dichas modificaciones, por los gastos que implican y por la imposibilidad de obtener retornos sobre las mismas. Pero si la apropiabilidad es muy alta, sólo una parte reducida de los beneficios de las modificaciones se extendería por todo el sistema económico, lo que brinda ventajas competitivas a las empresas que las hacen (Dosi, 1988b). De ahí las diversas discusiones en cuanto al alcance de los derechos de dominio sobre los productos tecnológicos y sus efectos en la competencia dentro de los mercados.

El tercer elemento del régimen tecnológico hace referencia a los patrones de demanda que enfrentan las empresas, los cuales determinan también los incentivos que existen para realizar modificaciones a cada tecnología. Por ejemplo, si la elasticidad precio de la demanda que enfrenta el producto de la empresa es alta, existen mayores incentivos para realizar modificaciones en los procesos y buscar eficiencia en costos, ya que cualquier variación en el precio puede perjudicar la empresa. Por otra parte, si la empresa enfrenta una elasticidad precio de la demanda baja, tiene mayores incentivos para explotar oportunidades tecnológicas que lleven a nuevos productos con nuevos precios, ya que los consumidores soportan mejor estas modificaciones (Urraca, 2008).

En definitiva, los cambios en el tamaño y crecimiento del mercado y en los precios relativos son factores influyentes en la tasa y la dirección del progreso técnico, así como en la selección de potenciales paradigmas a ser explorados por las empresas. No obstante, las condiciones de demanda no son las más importantes para el análisis del cambio técnico, ya que sólo son uno de los varios factores que determinan en qué componente del paradigma tecnológico la empresa debe fijar sus esfuerzos (Dosi, 1988a).

\section{LA EMPRESA Y LOS APRENDIZAJES}

Las empresas son unidades dinámicas con una organización y están enmarcadas en un paradigma tecnológico. Dado que son unidades en constante movimiento e inmersas en estados del mundo cambiantes, las empresas tienen la necesidad de contar con rutinas de aprendizajes. Es decir, las firmas pueden definirse, además, como organizaciones o unidades de aprendizaje para la adquisición, acumulación y generación de conocimiento y esta característica es parte misma de su tecnología (Malerba, 1992).

En efecto, las empresas cuentan con redes y vínculos de información interna y externa que afectan su comportamiento (Freeman, 1994). Como resultado, en las empresas tienen lugar múltiples procesos de aprendizaje y dentro de éstas se crea y reside una gran cantidad de conocimiento como resultado del historial de producción (Stiglitz y Greenwald, 2015). Es decir, cuando una empresa es liquidada, no sólo se pierden los insumos (trabajo y capital) conforme asume la visión neoclásica de la firma. Cuando termina una empresa, se pierden experticias, sincronizaciones, conocimiento creado sobre la configuración de los recursos y potencialidades para resolver problemas propios de cada paradigma tecnológico.

El aprendizaje de las empresas ha sido considerando como uno de los recursos y procesos más importantes dentro de las industrias (Malerba, 1992). Gran parte del aumento de la 
productividad ocurre cuando las empresas aprenden unas de otras y cuando la tecnología mejora a través de la práctica. Por esta razón, se ha sostenido que uno de los avances en las economías modernas ha sido la mejora en los procesos de aprendizaje, es decir, que las empresas hayan aprendido a aprender (Stiglitz y Greenwald, 2015).

Los métodos de aprendizaje son variados y las empresas pueden aprender de diferentes maneras. No se trata de procesos automáticos, sino de actividades conscientes y deliberadas que motivan el aprendizaje y que están vinculadas a diferentes fuentes de conocimiento internas y externas (Malerba, 1992).

De acuerdo con las fuentes y los tipos de conocimiento, las empresas pueden aprender haciendo (en la actividad interna de producción); aprender usando (utilizando los productos e insumos internos); aprender de los avances en ciencia y tecnología (absorbiendo los nuevos desarrollos externos); aprender de los derrames dentro de la industria (de lo que hacen los competidores y otras firmas); aprender interactuando (en la relación con fuentes ascendentes o descendentes externas del proceso de producción), y aprender buscando (mediante actividades internas de generación de nuevo conocimiento) (Malerba, 1992; Freeman, 1994).

Los diversos procesos de aprendizaje producen mejoras en el stock de conocimiento y en las capacidades tecnológicas de las empresas, lo cual genera una gama de trayectorias para el avance tecnológico (Malerba, 1992). En este proceso interactivo, los flujos de información y de conocimiento son importantes para comprender el comportamiento de las firmas y, por consiguiente, el desempeño de la economía (Freeman, 1994).

Al contrario de esta visión de la empresa, la teoría neoclásica considera que la empresa no aprende o no tiene necesidad de aprender. La firma se concibe como un receptor pasivo, que sólo tiene la capacidad de adquirir la información externa que se encuentra en un stock común y público. En esa medida, no debe llevar a cabo ningún proceso interactivo para la acumulación de conocimiento, pues este ya está a su disposición (Freeman, 1994). Así, esta teoría ignora el aprendizaje y resta importancia a las actividades de I+D, ya que asume que todas las firmas emplean las mejores prácticas y, por ende, no tienen nada que aprender (Stiglitz y Greenwald, 2015).

De acuerdo con el modelo neoclásico, la tecnología o el conocimiento del que disponen las empresas a priori se resume en una función de producción que mapea con exactitud cuánta cantidad de producto se consigue con ciertos insumos. Esta función resume toda la información relevante para las actividades de transformación de insumos, por lo que toda la atención gira sobre el proceso de optimización de dicha función. Este proceso se resuelve de manera mecánica una vez se cuenta con los parámetros del problema, los precios y la forma de la función de producción, los cuales solucionan el problema de optimización una vez en el tiempo. En consecuencia, la teoría neoclásica se presenta como una visión limitada, ya que la optimización no alcanza nunca un máximo en firmas que tienen procesos de aprendizaje de manera continua por el contacto con sus clientes, con el mercado, con sus procesos y con la ciencia misma. 
El aprendizaje no se puede esquematizar en términos simples como una serie de conjuntos de elecciones y resultados limitados y conocidos o como un simple parámetro que altera la función de producción que eleva la productividad marginal por insumo (Malerba, 1992). Contrario a esto, el aprendizaje es un fenómeno histórico, complejo e institucional que cada firma construye de forma idiosincrática y que se convierte en parte distintiva de la misma (Dosi, 1982).

Es posible afirmar entonces que el concepto de tecnología en sí también debe ser entendido como las rutinas de aprendizaje de las empresas, debido a la naturaleza intrínsecamente incierta que implica toda mejora en los procesos de producción. En este sentido, la limitación del concepto de función de producción reside en que carece de la conceptualización de la tecnología per se y no proporciona un marco adecuado para el análisis y medición del cambio tecnológico (Sahal, 1981). En suma, la economía neoclásica tiene una concepción corta de la tecnología, al concebirla como una caja negra sin esquema organizativo ni aprendizajes (Dosi, 1982).

Con el fin de superar las falencias del modelo neoclásico y de conjugar los elementos esenciales para la conceptualización integral de la empresa y de la tecnología expuestos antes, se presenta a continuación una esquematización alterna de la firma en la figura 3.

\section{Paradigma tecnológico}

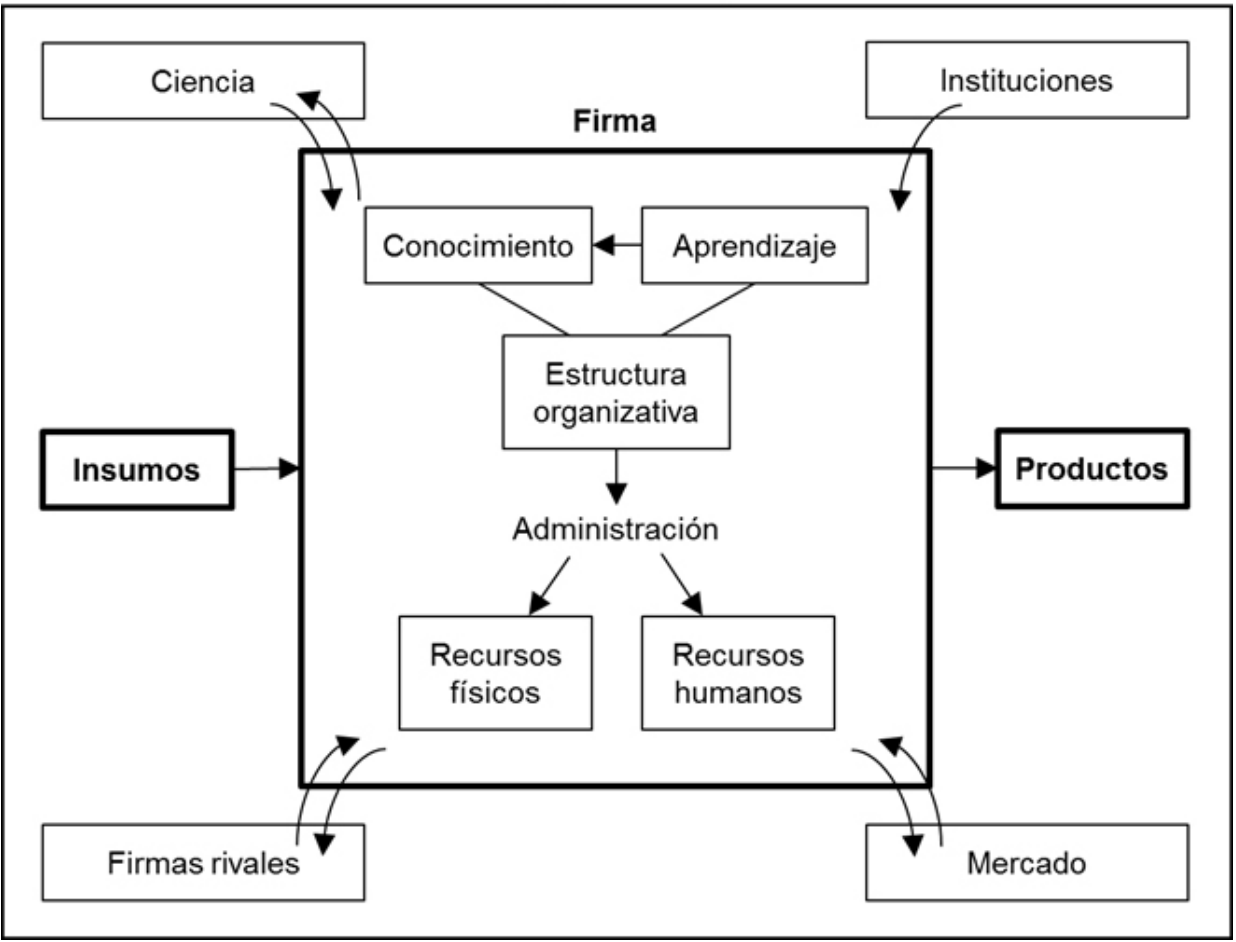

Figura 3. La firma como organización de aprendizaje en un paradigma tecnológico. Elaboración propia. 
La propuesta que se presenta busca focalizar el hecho de que la firma debe ser concebida como una organización administrativa de recursos productivos, cuya actividad económica se desarrolla dentro de un esquema organizativo y una planificación deliberada (Penrose, 2009). Además, la empresa debe enfrentar y está enmarcada en un paradigma tecnológico que configuran unas relaciones únicas con la ciencia (oportunidad tecnológica), las instituciones (condiciones de apropiabilidad) y el mercado (condiciones de demanda), las cuales dependen de la tecnología específica de cada firma (Dosi, 1982).

La firma debe entenderse también como una unidad de aprendizaje para la adquisición, acumulación y generación de conocimiento, por lo cual es dable concluir que la tecnología en sí misma involucra también este aprendizaje, el cual afecta el conocimiento de la firma, su organización y su administración (Malerba, 1992). En este sentido, con las visiones alternativas referenciadas es posible configurar, en conjunto, una conceptualización más integral de la empresa y de su tecnología, lo que permite también visibilizar mejor su relevancia en las economías de mercado.

\section{CONCLUSIONES}

El análisis de la firma en economías de mercado requiere de nociones que trascienden las asunciones limitadas de la teoría neoclásica. Por lo tanto, se hace necesario considerar otras visiones de la firma y la tecnología, que vayan más allá de los conceptos neoclásicos de 'caja negra' transformadora de insumos, por medio de información pública, simétrica, completa y perfecta.

Tales visiones hacen referencia a las teorías de estructura organizativa, paradigmas tecnológicos y aprendizaje organizacional, las cuales fueron descritas y analizadas en precedencia. Cada una de estas aproximaciones teóricas postula elementos conceptuales esenciales para comprender la firma y la tecnología en la actualidad. Sin embargo, su alcance es insuficiente para explicar la complejidad actual de estos fenómenos.

Ante estas limitaciones, en este documento se expuso una visión alterna de la firma y de la tecnología, que integra los postulados relevantes de las perspectivas aludidas para proponer una conceptualización integral de estos fenómenos. Así, se pudo establecer que la firma debe ser concebida como una organización administrativa de recursos productivos y una unidad de aprendizaje, que se enmarca en un determinado paradigma tecnológico. Por su parte, se planteó que la tecnología de la empresa es dinámica y que involucra organización y aprendizajes particulares y dependientes del paradigma o régimen en que esta desarrolla su producción.

\section{REFERENCIAS BIBLIOGRÁFICAS}

Arrow, K. y Debreu, G. (1954). Existence of equilibrium for a competitive Economy. Econometrica, 22, 265-290.

Dosi, G. (1982). Technological paradigms and technological trajectories. A suggested interpretation of the determinants and directions of technical change. Research Policy, 11, 147-162. 
Dosi, G. (1988a). Sources, procedures, and microeconomic effects of innovation. Journal of Economic Literature, 26, 1120-1171.

Dosi, G. (1988b). The nature of the innovative process. In G. Dosi, C. Freeman, R. Nelson, G. Silverberg, \& L. Soete (Eds.), Technical change and economic theory (pp. 221-238). London \& New York: Pinter Publisher.

Freeman, C. (1994). The economics of technical change. Cambridge Journal of Economics, 18(5), 463514.

Heijs, J. \& Buesa, M. (2016). Manual de economía de innovación. Tomo I. Teoría del cambio tecnológico y sistemas nacionales de innovación. Madrid: Universidad Complutense Madrid.

Kuhn, T. (1970). The Structure of Scientific Revolutions. 2a ed. Chicago: University of Chicago Press.

Malerba, F. (1992). Learning by firms and incremental technical change. The Economic Journal, 102(413), 845-859.

Monsalve, S. (1999). Introducción a los conceptos de equilibrio en economía. Editorial Universidad Nacional: Bogotá.

Nelson, R. (1988). [Preface]. In G. Dosi, C. Freeman, R. Nelson, G. Silverberg, \& L. Soete (Eds.), Technical change and economic theory (pp. 219-220). London \& New York: Pinter Publisher.

Penrose, E. (2009). The theory of the growth of the firm. 4a ed. New York: Oxford University Press.

Sahal, D. (1981). Alternative conceptions of technology. Research Policy, 10, 2-24.

Sala-i-Martin, X. (2000). Apuntes de crecimiento económico. Madrid: Antoni Bosch Editor.

Stiglitz, J. (2000). The Contributions of the Economics of Information to Twentieth Century Economics. The Quarterly Journal of Economics, 115(4), 1441-1478.

Stiglitz, J. E. \& Greenwald, B. C. (2015). Creating a learning society. A new approach to growth, development, and social progress. New York: Columbia University Press.

Urraca, A. (2008). Persistência versus mudança estrutural da especialização tecnológica do Brasil. Economia e Sociedade, 17 (3) (34), 403-427.

Varian, H. R. (1992). Análisis microeconómico. (María Esther Rabasco y Luis Toharia, trad.). 3a ed. Antoni Bosch. (Obra original publicada en 1978)

\section{Para citaciones:}

Galvis, J. y Echavarría, M. (2018). Una visión alterna de la firma desde la organización, el aprendizaje y los paradigmas tecnológicos. Panorama Económico, 26(4), 481-496. 
JUAN CAMILO GALVIS-CIRO, MARÍA ALEJANDRA ECHAVARRÍA-ARCILA

Una visión alterna de la firma desde la organización, el aprendizaje y los paradigmas tecnológicos

\section{AUTORES}

Juan Camilo Galvis-Ciro

María Alejandra Echavarría-Arcila 